\title{
Structure Formation in Anisotropic Disks
}

\author{
Eduard Vorobyov ${ }^{1}$ and Christian Theis ${ }^{2}$ \\ ${ }^{1}$ Dept. of Physics and Astronomy, Univ. of Western Ontario, London, Canada, \\ email: eduard_vorobev@mail.ru \\ ${ }^{2}$ Institute of Astronomy, Univ. of Vienna, Austria, email: theis@astro.univie.ac.at
}

The majority of normal disk galaxies are characterized by non-axisymmetric structures like spirals or bars. These structural elements have been widely discussed in the literature as a result of gravitational instabilities which are connected to growing density waves or global instabilities of disks. A first insight into the properties of galactic discs was provided by linear stability analysis. However, a disadvantage of linear stability analysis remained its restriction to small perturbations, both in amplitude and wavelength. Thus, numerical simulations, especially hydrodynamical and stellar-hydrodynamical simulations became a primary tool for the analysis of galactic evolution.

Here, we present our Boltzmann moment equation approach for the dynamics of stars (BEADS-2D), which is a finite-difference Eulerian numerical code designed for the modelling of anisotropic and non-axisymmetric flat stellar disks. The BEADS-2D code solves the Boltzmann moment equations up to second order in the thin-disk approximation including the self-gravity of the disk. This allows us to obtain the anisotropy of the velocity ellipsoid and the vertex deviation in the plane of the disk.

We study the time-dependent evolution of exponential stellar disks in the linear regime and beyond. The disks are initially characterized by different values of the Toomre parameter and they are embedded in a dark matter halo, yielding a rotation curve composed of a rigid central part and a flat outer region. Starting from a near equilibrium state, several unstable modes develop in the disk.

We found the following main results:

- The additional degrees of freedom added by an anisotropic velocity distribution destabilize galactic disks strongly. Such disks are much more unstable than the corresponding isotropic disks (i.e. either gaseous disks or stiff polytropic disks which are often used to mimic stellar disks).

- Stability is reached for a Toomre parameter of about 3.15 which is in agreement with predictions of linear stability analysis for stellar disks.

- Lopsided modes become more important for increased Toomre parameter.

- Considerable vertex deviations are found in regions with strongly perturbed mass distributions, i.e. near the spiral arms. The vertex deviations are especially large at the convex edge of the spiral arms, whereas they are small at the concave edge.

- The mean vertex deviations correlate well with the global Fourier amplitudes, reaching mean values of about $12^{\circ}-20^{\circ}$ in the saturation stage. Local values of the vertex deviation can reach up to almost $90^{\circ}$.

Vertex deviations are strongly influenced by a non-axisymmetric potential.

- Near the convex edge of the spiral arms, the ratio of radial to azimuthal components of the velocity ellipsoid can deviate considerably from the values predicted from the epicycle approximation $\left(\sigma_{\phi \phi} / \sigma_{r r}<0.4\right)$.

\section{Acknowledgements}

CT is grateful for financial support by the IAU. 